\title{
Dirichlet Problem for Complex Poisson Equation in a Half Hexagon Domain
}

\author{
Bibinur Shupeyeva \\ Nazarbayev University, 53 Kabanbay Batyr Avenue, Astana 010000, Kazakhstan \\ Correspondence should be addressed to Bibinur Shupeyeva; bibinur.shupeyeva@nu.edu.kz
}

Received 21 October 2015; Revised 15 December 2015; Accepted 10 January 2016

Academic Editor: Vladislav Kravchenko

Copyright (C) 2016 Bibinur Shupeyeva. This is an open access article distributed under the Creative Commons Attribution License, which permits unrestricted use, distribution, and reproduction in any medium, provided the original work is properly cited.

The parqueting-reflection method is applied to a nonregular domain and the harmonic Green function for the half hexagon is constructed. The related Dirichlet problem for the Poisson equation is solved explicitly.

\section{Introduction}

The basic boundary value problems for the second-order complex partial differential equations are the harmonic Dirichlet and Neumann problems for the Laplace and Poisson equations. In order to find the solution in explicit or closed form diverse methods have been applied. In case a given domain $D$ is simply connected and has a piecewise smooth boundary $\partial D$ the tools of complex analysis such as Schwarz reflection principle and conformal mapping serve perfectly. When a given domain $D$ is piecewise smooth polygonal and has corners the Schwarz-Christoffel formula can be used. Difficulties arise since the elliptic integrals appearing in the formula imply complicated computations and need to be solved numerically. As analogue to this formula, another method can be applied which gives the covering of the entire complex plane $\mathbb{C}$ by reflection of the given domain $D$ at its boundary. The method is fully described in numerous papers of Begehr and other authors; see, for example [1-12]. Our aim is to find the solution of the Dirichlet boundary value problem for the Poisson equation through the Poisson integral formula. It is known that the Poisson kernel function is an analogue of the Cauchy kernel for the analytic functions and the Poisson integral formula solves the Dirichlet problem for the inhomogeneous Laplace equation. One way to obtain the Poisson kernel leads to the harmonic Green function which is to be constructed by use of the parqueting-reflection method.
In this paper we first consider the half hexagon domain and implement the parqueting-reflection method. The reflection points treated in a proper way help to construct the certain meromorphic functions needed to find the harmonic Green function and representation formula. The later one provides the solution to the harmonic Dirichlet problem which is shown in the last part.

\section{Half Hexagon Domain and Poisson Kernel}

We consider a polygonal domain with corner points. The half hexagon denoted as $P^{+}$with four corner points at $2,1+i \sqrt{3}$, $-1+i \sqrt{3}$, and -2 lies in the upper half plane. A point $z \in P^{+}$ will later serve as a pole of the Green function. Its complex conjugate $\bar{z}$ does not lie in $P^{+}$. $P^{+}$is reflected at the real axis so that the entire hexagon $P$ (Figure 1 ) is obtained. The pole $z$ is reflected onto $\bar{z}$ which will later become a zero of a certain meromorphic function related to the Green function. The points $z$ and $\bar{z}$ from $P$ are reflected again through all the sides of the hexagon, starting with the right upper side and continuing in a positive direction. The successive reflections of $z$ give the points, which will later become zeros of the meromorphic function mentioned above. They are

$$
\begin{gathered}
-\frac{1}{2}(1+i \sqrt{3}) \bar{z}+3+i \sqrt{3}, \\
\bar{z}+2 i \sqrt{3},
\end{gathered}
$$




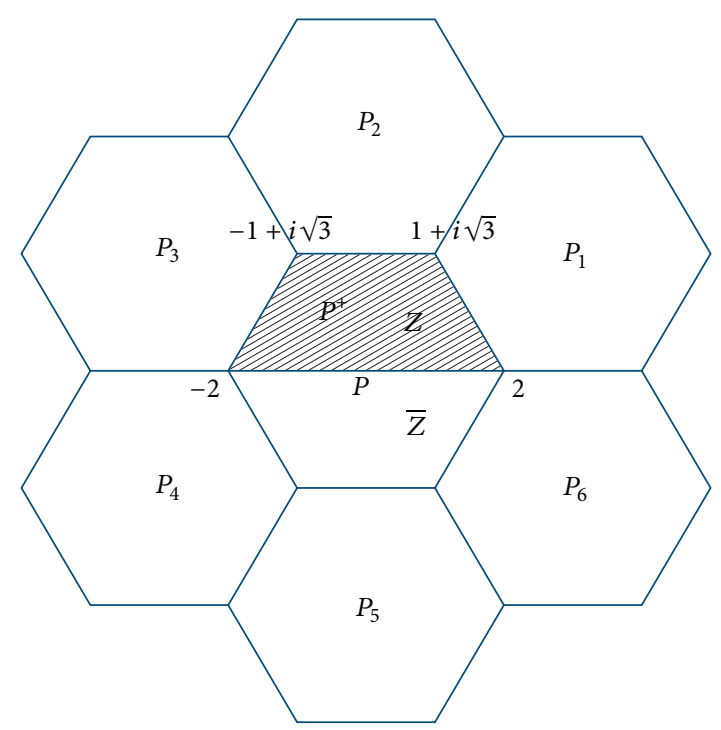

Figure 1: Hexagons.

$$
\begin{gathered}
-\frac{1}{2}(1-i \sqrt{3}) \bar{z}-3+i \sqrt{3} \\
-\frac{1}{2}(1+i \sqrt{3}) \bar{z}-3-i \sqrt{3} \\
\bar{z}-2 i \sqrt{3} \\
-\frac{1}{2}(1-i \sqrt{3}) \bar{z}+3-i \sqrt{3}
\end{gathered}
$$

Reflection of the point $\bar{z} \in P$ defines the poles of the meromorphic function in the hexagons $P_{1}, \ldots, P_{6}$. These points in turn are reflected through the sides of the new hexagons, except for reflecting to the original hexagon $P$. Hence each hexagon includes now 3 poles and 3 zeros. Continuation of these operations reveals that all the points have the same coefficients of rotation: $1,-(1 / 2)(1+i \sqrt{3})$, $-(1 / 2)(1-i \sqrt{3})$, and displacement $3 m+i \sqrt{3} n, m+n \in 2 \mathbb{Z}$. Note that reflection includes rotation and shifting and the points from one hexagon can be expressed through the points of another one. In general the points from the hexagons differ by displacements $6 m$ in the direction of the real and $2 i \sqrt{3} n$ in the direction of the imaginary axes. Thus the main period is $\mu_{m n}=6 m+2 i \sqrt{3} n$.

Obviously, the repeated reflections of the point $z \in P^{+}$are representable in different ways, using either of the points

$$
\begin{aligned}
& z_{1}=-\frac{1}{2}(1+i \sqrt{3}) \bar{z}+3+i \sqrt{3}, \\
& z_{2}=-\frac{1}{2}(1+i \sqrt{3}) z+3+i \sqrt{3} \text { or } \\
& \check{z}_{1}=-\frac{1}{2}(1-i \sqrt{3}) \bar{z}-3+i \sqrt{3}, \\
& \check{z}_{2}=-\frac{1}{2}(1-i \sqrt{3}) z-3+i \sqrt{3},
\end{aligned}
$$

which are connected by the relations $\overline{\check{z}}_{2}=z_{1}-6-2 i \sqrt{3}$ and $\overline{z_{1}}=z_{2}-6-2 i \sqrt{3}$.

In general, all reflection points are either given by

$$
\begin{gathered}
z+\omega_{m n} \\
\bar{z}_{1}+\omega_{m n}, \\
z_{2}+\omega_{m n} \\
\bar{z}+\omega_{m n} \\
z_{1}+\omega_{m n} \\
\bar{z}_{2}+\omega_{m n}
\end{gathered}
$$

or by

$$
\begin{aligned}
& z+\omega_{m n}, \\
& \check{z}_{1}+\omega_{m n}, \\
& \check{z}_{2}+\omega_{m n}, \\
& \bar{z}+\omega_{m n}, \\
& \check{z}_{1}+\omega_{m n}, \\
& \check{z}_{2}+\omega_{m n},
\end{aligned}
$$

where $\omega_{m n}=3 m+i \sqrt{3} n$ such that $m+n \in 2 \mathbb{Z}$.

We choose zeros as direct reflection of poles and poles as direct reflection of zeros. Then having a set of zeros and a set of poles, one can construct the Schwarz kernel for $\mathrm{P}^{+}$and treat the related Schwarz problem [9] and Riemann-Hilbert-type boundary value problem.

The half hexagon can be viewed as the complement of the intersection of four half planes. We define them by $H_{1}^{-}$being the right-hand half plane which has the boundary line passing through the points 2 and $1+i \sqrt{3}, H_{2}^{-}$being the upper half 
plane with the border line through the points $\pm 1+i \sqrt{3}, H_{3}^{-}$ being the left-hand half plane with the border line passing through the points $-1+i \sqrt{3}$ and -2 , and $H_{4}^{-}$being the half plane which is below the real axis.

Let then $\mathrm{H}_{1}^{+}, \mathrm{H}_{2}^{+}, \mathrm{H}_{3}^{+}, \mathrm{H}_{4}^{+}$be the complementary half planes of those listed above. The Green functions of these half planes are, in fact, the Green functions for the complementary half planes $H_{1}^{-}, \ldots, H_{4}^{-}$. The outward normal derivatives of the Green function on the boundary is the Poisson kernel. The kernel provides the boundary condition $w=\gamma$ in the Dirichlet problem.

The Poisson kernels can be found from the respective Green functions $G_{1}(z, \zeta), z=x+i y, \zeta=\xi+i \eta$ as described below.

For the half plane $H_{1}^{+}$with the boundary described by the relation $\zeta-2=-(1 / 2)(1+i \sqrt{3})(\bar{\zeta}-2)$ we have

$$
\begin{array}{r}
G_{1}(z, \zeta)=\log \left|\frac{(1 / 2)(1+i \sqrt{3})(\bar{\zeta}-2)+z-2}{\zeta-z}\right|^{2}, \\
z, \zeta \in H_{1}^{+}, \\
-\frac{1}{2} \partial_{\nu_{\zeta}} G_{1}(z, \zeta)=-\frac{(\sqrt{3}-i)}{4} \frac{z-z_{1}}{|\zeta-z|^{2}}, \\
\zeta \in \partial H_{1}^{+}, \quad z \in H_{1}^{+},
\end{array}
$$

where $z_{1}=-(1 / 2)(1+i \sqrt{3}) \bar{z}+3+i \sqrt{3}, z_{1} \in H_{1}^{+}$.

For the half plane $H_{2}^{+}$the relation on the boundary is given as $\zeta=\bar{\zeta}+2 i \sqrt{3}$; then

$$
\begin{array}{r}
G_{1}(z, \zeta)=\log \left|\frac{\bar{\zeta}-z+2 i \sqrt{3}}{\zeta-z}\right|^{2}, \quad z, \zeta \in H_{2}^{+}, \\
-\frac{1}{2} \partial_{\nu_{\zeta}} G_{1}(z, \zeta)=-\frac{1}{i} \frac{z-z_{2}}{|\zeta-z|^{2}}, \quad \zeta \in \partial H_{2}^{+}, \quad z \in H_{2}^{+} ;
\end{array}
$$

here $z_{2}=\bar{z}+2 i \sqrt{3}, z \in H_{2}^{+}$.

The boundary of the half plane $\mathrm{H}_{3}^{+}$is described by $\zeta+2=$ $-(1 / 2)(1-i \sqrt{3})(\bar{\zeta}+2)$ and

$$
\begin{array}{r}
G_{1}(z, \zeta)=\log \left|\frac{(1 / 2)(1-i \sqrt{3})(\bar{\zeta}+2)+z+2}{\zeta-z}\right|^{2}, \\
z, \zeta \in H_{3}^{+}, \\
-\frac{1}{2} \partial_{\nu_{\zeta}} G_{1}(z, \zeta)=-\frac{\sqrt{3}+i}{4} \frac{z-\check{z_{1}}}{|\zeta-z|^{2}}, \\
\zeta \in \partial H_{3}^{+}, z \in H_{3}^{+},
\end{array}
$$

where $\check{z}_{1}=-(1 / 2)(1-i \sqrt{3}) \bar{z}-3+i \sqrt{3}, z \in H_{3}^{+}$.
Finally, for the half plane $H_{4}^{+}$with the boundary described by $\zeta=\bar{\zeta}$, we have

$$
\begin{gathered}
G_{1}(z, \zeta)=\log \left|\frac{\bar{\zeta}-z}{\zeta-z}\right|^{2}, \quad z, \zeta \in H_{4}^{+}, \\
-\frac{1}{2} \partial_{\nu_{\zeta}} G_{1}(z, \zeta)=\frac{1}{i} \frac{z-\bar{z}}{|\zeta-z|^{2}}, \quad \zeta \in \partial H_{4}^{+}, \quad z \in H_{4}^{+} .
\end{gathered}
$$

\section{Green Representation Formula}

The method of reflections helps to find the harmonic Green function; see [3-5]. The reflection points given in (3) or (4) are used to construct a meromorphic function:

$$
\begin{aligned}
& B_{1}(z, \zeta) \\
& =\prod_{m+n \in 2 \mathbb{Z}} \frac{\left(\zeta-\bar{z}-\omega_{m n}\right)\left(\zeta-z_{1}-\omega_{m n}\right)\left(\zeta-\overline{z_{2}}-\omega_{m n}\right)}{\left(\zeta-\omega_{m n}\right)\left(\zeta-\overline{z_{1}}-\omega_{m n}\right)\left(\zeta-z_{2}-\omega_{m n}\right)} \\
& =\prod_{m+n \in 2 \mathbb{Z}} \frac{\left(\zeta-\omega_{m n}-2\right)^{3}-(\bar{z}-2)^{3}}{\left(\zeta-\omega_{m n}-2\right)^{3}-(z-2)^{3}}
\end{aligned}
$$

where $z_{1}=-(1 / 2)(1+i \sqrt{3}) \bar{z}+3+i \sqrt{3}, z_{2}=-(1 / 2)(1+i \sqrt{3}) z+$ $3+i \sqrt{3}$, or a function

$$
B_{2}(z, \zeta)=\prod_{m+n \in 2 \mathbb{Z}} \frac{\left(\zeta-\omega_{m n}+2\right)^{3}-(\bar{z}+2)^{3}}{\left(\zeta-\omega_{m n}+2\right)^{3}-(z+2)^{3}},
$$

where $\check{z}_{1}=-(1 / 2)(1-i \sqrt{3}) \bar{z}-3+i \sqrt{3}, \check{z}_{2}=-(1 / 2)(1-i \sqrt{3}) z-$ $3+i \sqrt{3}$. Here $z$ is considered as a parameter and $\zeta \in \mathbb{C}$ is the variable.

For the boundary part $\partial_{2} P$, the line from $1+i \sqrt{3}$ to $-1+i \sqrt{3}$, a meromorphic function $B_{3}(z, \zeta)$, is deduced from $B_{1}(z, \zeta)$ by rotating the variable $\zeta$ and the parameter $z$ about the angle $\pi / 3$ :

$$
\begin{aligned}
B_{1} & \left(-\frac{1}{2}(1+i \sqrt{3}) z,-\frac{1}{2}(1+i \sqrt{3}) \zeta\right) \\
= & \prod_{m+n \in 2 \mathbb{Z}} \frac{\left(\zeta-\omega_{m n}+1-i \sqrt{3}\right)^{3}-(\bar{z}+1+i \sqrt{3})^{3}}{(\zeta n+1-i \sqrt{3})^{3}-(z+1-i \sqrt{3})^{3}},
\end{aligned}
$$

which becomes 1 on the boundary $\partial_{2} P$, where $\zeta-i \sqrt{3}=\bar{\zeta}+$ $i \sqrt{3}$.

The following lemmas will be needed to prove the Green representation formula below. The complete proofs of these lemmas are given in [9].

Lemma 1. The infinite product

$$
\prod_{m+n \in 2 \mathbb{Z}} \frac{\left(\zeta-\omega_{m n}-2\right)^{3}-(\bar{z}-2)^{3}}{\left(\zeta-\omega_{m n}-2\right)^{3}-(z-2)^{3}}
$$

converges, where $\omega_{m n}=3 m+i \sqrt{3} n, m+n \in 2 \mathbb{Z}$. 
Lemma 2. The equalities $B_{1}(z, \zeta)=B_{2}(z, \zeta)=B_{3}(z, \zeta)$ hold for $(z, \zeta) \in P^{+} \times \partial P^{+}$.

The proof of this equality is based on the fact that the functions $B_{1}(z, \cdot), B_{2}(z, \cdot)$, since $B_{3}(z, \cdot)$ can be obtained from $B_{1}(z, \cdot)$, have the same poles and zeros; see [9].

The Green function must satisfy the following conditions; see $[13]$ :

$\left(1^{0}\right) G_{1}(z, \zeta)$ is harmonic in $P^{+} \backslash\{z\}$;

$\left(2^{0}\right) G_{1}(z, \zeta)+\log |\zeta-z|^{2}$ is harmonic in $\zeta \in P^{+}$for any $z \in P^{+}$

$\left(3^{0}\right) \lim _{\zeta \rightarrow \partial P^{+}} G(z, \zeta)=0$ for any $z \in P^{+} ;$

and the additional properties:

$\left(4^{0}\right) G_{1}(z, \zeta)=G_{1}(\zeta, z), z$ and $\zeta$ in $P^{+}, z \neq \zeta$;

$\left(5^{0}\right) G_{1}(z, \zeta)>0, z$ and $\zeta$ in $P^{+}, z \neq \zeta$.

By the properties $\left(1^{0}\right)-\left(3^{0}\right)$ the Green function $G_{1}(z, \zeta)$ is uniquely defined. Obviously, $G_{1}(z, \zeta)$ as defined above is harmonic in $\zeta \in P^{+} \backslash\{z\}$ as $B_{1}(z, \zeta)$ is analytic in $P^{+}$up to a single pole at $z$. Adding $\log |\zeta-z|^{2}$ gives a harmonic function of $\zeta \in P^{+}$. The symmetry property $\left(4^{0}\right)$ is a consequence from the properties $\left(1^{0}\right)-\left(3^{0}\right)$. The harmonic Green function for the half hexagon $P^{+}$is

$$
\begin{aligned}
G_{1}(z, \zeta) & =\log \left|B_{1}(z, \zeta)\right|^{2}=\log \left|B_{2}(z, \zeta)\right|^{2} \\
& =\log \left|B_{3}(z, \zeta)\right|^{2}
\end{aligned}
$$

or, by the symmetry property,

$$
\begin{aligned}
& G_{1}(z, \zeta)=\log \left|\prod_{m+n \in 2 \mathbb{Z}} \frac{\left(z-\omega_{m n}-2\right)^{3}-(\bar{\zeta}-2)^{3}}{\left(z-\omega_{m n}-2\right)^{3}-(\zeta-2)^{3}}\right|^{2}, \\
& G_{1}(z, \zeta)=\log \left|\prod_{m+n \in 2 \mathbb{Z}} \frac{\left(z-\omega_{m n}+2\right)^{3}-(\bar{\zeta}+2)^{3}}{\left(z-\omega_{m n}+2\right)^{3}-(\zeta+2)^{3}}\right|^{2}, \\
& G_{1}(z, \zeta) \\
& =\log \left|\prod_{m+n \in 2 \mathbb{Z}} \frac{\left(z-\omega_{m n}+1-i \sqrt{3}\right)^{3}-(\bar{\zeta}+1+i \sqrt{3})^{3}}{\left(z-\omega_{m n}+1-i \sqrt{3}\right)^{3}-(\zeta+1-i \sqrt{3})^{3}}\right|^{2} .
\end{aligned}
$$

Lemma 3. The function $G_{1}(z, \zeta)$ has vanishing boundary values on $\partial P^{+}$; that is,

$$
\lim _{\zeta \rightarrow \zeta_{0} \in \partial P^{+}} G_{1}(z, \zeta)=0 .
$$

Theorem 4 (see [13]). Anyw $\in C^{2}\left(P^{+} ; \mathbb{C}\right) \cap C^{1}\left(\overline{P^{+}} ; \mathbb{C}\right)$ can be represented as

$$
\begin{aligned}
w(z)= & -\frac{1}{4 \pi} \int_{\partial P^{+}} w(\zeta) \partial_{v_{\zeta}} G_{1}(z, \zeta) d s_{\zeta} \\
& -\frac{1}{\pi} \int_{P^{+}} w_{\zeta \bar{\zeta}}(\zeta) G_{1}(z, \zeta) d \xi d \eta,
\end{aligned}
$$

where $s_{\zeta}$ is the arc length parameter on $\partial P^{+}$with respect to the variable $\zeta=\xi+$ in and $G_{1}(z, \zeta)=2 G(z, \zeta)$ is the harmonic Green function for $P^{+}$.
We consider now the different forms of the Green function and take the derivatives $\partial_{\zeta} G_{1}(z, \zeta), \partial_{\bar{\zeta}} G_{1}(z, \zeta)$.

For the right-hand side, a boundary $\partial_{1} P^{+}$, we choose the form (14) for $\zeta \in \partial P^{+}, z \in P^{+}$. Here the outward normal derivative is $\partial_{\nu_{\zeta}}=(\sqrt{3} / 2+i / 2) \partial_{\zeta}+(\sqrt{3} / 2-i / 2) \partial_{\bar{\zeta}}$; then

$$
\begin{aligned}
& \partial_{\nu_{\zeta}} G_{1}(z, \zeta)=-3(\sqrt{3}+i)(\zeta-2)^{2} \\
& \cdot \sum_{m+n \in 2 \mathbb{Z}} \frac{\left(z-\omega_{m n}-2\right)^{3}-\left(\overline{z-\omega_{m n}-2}\right)^{3}}{\left|\left(z-\omega_{m n}-2\right)^{3}-(\zeta-2)^{3}\right|^{2}},
\end{aligned}
$$

since $\zeta-2=-(1 / 2)(1+i \sqrt{3})(\bar{\zeta}-2),(\zeta-2)^{3}=(\bar{\zeta}-2)^{3}$.

For the boundary part $\partial_{4} P^{+}$, a line between $(-2,0),(2,0)$ on a real axis, the outward normal derivative is $\partial_{\nu_{\zeta}}=-i\left(\partial_{\zeta}-\right.$ $\left.\partial_{\bar{\zeta}}\right), \zeta=\bar{\zeta}$; then

$$
\begin{aligned}
& \partial_{\nu_{\zeta}} G_{1}(z, \zeta)=6 i(\zeta-2)^{2} \\
& \cdot \sum_{m+n \in 2 \mathbb{Z}} \frac{\left(z-\omega_{m n}-2\right)^{3}-\left(\overline{z-\omega_{m n}-2}\right)^{3}}{\left|\left(z-\omega_{m n}-2\right)^{3}-(\zeta-2)^{3}\right|^{2}} .
\end{aligned}
$$

For the boundary part $\partial_{3} P^{+}$on the left-hand side of $P^{+}$, we take form (15). The outward normal derivative is $\partial_{v_{\zeta}}=$ $(\sqrt{3} / 2-i / 2) \partial_{\zeta}+(\sqrt{3} / 2+i / 2) \partial_{\bar{\zeta}}$ also here $\zeta=\check{\zeta}_{1}=-(1 / 2)(1-$ $i \sqrt{3}) \bar{\zeta}-3+i \sqrt{3}$ and $(\zeta+2)^{3}=(\bar{\zeta}+2)^{3}$; then

$$
\begin{aligned}
& \partial_{v_{\zeta}} G_{1}(z, \zeta)=-3(\sqrt{3}-i)(\zeta+2)^{2} \\
& \cdot \sum_{m+n \in 2 \mathbb{Z}} \frac{\left(z-\omega_{m n}+2\right)^{3}-\left(\overline{z-\omega_{m n}+2}\right)^{3}}{\left|\left(z-\omega_{m n}+2\right)^{3}-(\zeta+2)^{3}\right|^{2}} .
\end{aligned}
$$

For the upper boundary part $\partial_{2} P^{+}$, a line joining the points $\pm 1+i \sqrt{3}$, form (16) is valid. Here $\partial_{\nu_{\zeta}}=i\left(\partial_{\zeta}-\partial_{\bar{\zeta}}\right)$ and $\zeta-i \sqrt{3}=$ $\bar{\zeta}+i \sqrt{3}$; then $\partial_{v_{\zeta}} G_{1}(z, \zeta)$ is

$$
\begin{aligned}
& -6 i(\zeta+1-i \sqrt{3})^{2} \\
& \cdot \sum_{m+n \in 2 \mathbb{Z}} \frac{\left(z-\omega_{m n}+1-i \sqrt{3}\right)^{3}-\left(\overline{z-\omega_{m n}+1-i \sqrt{3}}\right)^{3}}{\left|\left(z-\omega_{m n}+1-i \sqrt{3}\right)^{3}-(\zeta+1-i \sqrt{3})^{3}\right|^{2}} .
\end{aligned}
$$

\section{Harmonic Dirichlet Problem}

The representation formula in Theorem 4 provides the solution to the Dirichlet problem for the Poisson equation.

At first the boundary behavior of the integral is to be studied. Let for $\gamma \in C\left(\partial P^{+} ; \mathbb{R}\right)$

$$
\varphi(z)=-\frac{1}{4 \pi} \int_{\partial P^{+}} \gamma(\zeta) \partial_{\nu_{\zeta}} G(z, \zeta) d s_{\zeta}, \quad z \in P^{+} .
$$

Lemma 5. For $\gamma \in C\left(\partial P^{+} ; \mathbb{R}\right)$ the function presented in (23) satisfies the relation

$$
\lim _{z \rightarrow \zeta_{0}} \varphi(z)=\gamma\left(\zeta_{0}\right),
$$

where $\zeta_{0}$ is any fixed point on $\partial P^{+} \backslash\{ \pm 2, \pm 1+i \sqrt{3}\}$. 
Proof. Let $\zeta_{0}$ be defined on different boundary parts and consider the boundary behavior when $z \rightarrow \zeta_{0}$.

Case 1. If $\zeta_{0}$ is taken on $\partial_{1} P^{+}$so that $\zeta_{0}=-(1 / 2)(1+i \sqrt{3}) \overline{\zeta_{0}}+$ $3+i \sqrt{3}$ then

$$
\begin{aligned}
& \left(\zeta_{0}-2\right)^{2}=-\frac{1}{2}(1-i \sqrt{3})\left(\zeta_{0}-2\right)^{2}, \\
& \left(\zeta_{0}-2\right)^{3}=\left(\overline{\zeta_{0}}-2\right)^{3} .
\end{aligned}
$$

On $\partial_{1} P^{+}$where $\zeta=\zeta_{1}=-(1 / 2)(1+i \sqrt{3}) \bar{\zeta}+3+i \sqrt{3},(\zeta-2)^{3}=$ $(\bar{\zeta}-2)^{3}$.
For $m=n=0$ formula (19) gives

$$
\begin{aligned}
- & 3(\sqrt{3}+i)(\zeta-2)^{2} \frac{(z-2)^{3}-(\bar{z}-2)^{3}}{\left|(z-2)^{3}-(\zeta-2)^{3}\right|^{2}} \\
= & -3(\sqrt{3}+i)(\zeta-2)^{2} \\
& \cdot \frac{(z-\bar{z})\left[(z-2)^{2}+(z-2)\left(z_{1}-2\right)+\left(z_{1}-2\right)^{2}\right]}{|z-\zeta|^{2}\left|(z-2)^{2}+(z-2)(\zeta-2)+(\zeta-2)^{2}\right|^{2}} .
\end{aligned}
$$

Because $z_{1}=-(1 / 2)(1+i \sqrt{3}) \bar{z}+3+i \sqrt{3}$, then $\left(z_{1}-2\right)^{3}=$ $(\bar{z}-2)^{3}$. The limit in the following ratio as $z \rightarrow \zeta_{0}$ and $\zeta=\zeta_{0}$ gives

$$
\lim _{z \rightarrow \zeta_{0}}\left\{\frac{-3(\sqrt{3}+i)(\zeta-2)^{2}\left[(z-2)^{2}+(z-2)\left(z_{1}-2\right)+\left(z_{1}-2\right)^{2}\right]}{\left|(z-2)^{2}+(z-2)(\zeta-2)+(\zeta-2)^{2}\right|^{2}}\right\}=\frac{-(\sqrt{3}+i)\left(\zeta_{0}-2\right)^{4}}{\left|\zeta_{0}-2\right|^{4}}=(\sqrt{3}-i), \quad \zeta_{0} \neq 2 .
$$

For the other terms of the sum,

$$
\begin{aligned}
\left(z-\omega_{m n}-2\right)^{3} & =\left[-\frac{1}{2}(1-i \sqrt{3})\left(z-\omega_{m n}-2\right)\right]^{3} \\
& =\left(\overline{z_{1}}-\omega_{k l}-2\right)^{3}
\end{aligned}
$$

which follows from the rearrangement of the indices in $\omega_{m n}$ for certain $k+l \in 2 \mathbb{Z}$. Thus

$$
\begin{aligned}
& \sum_{\substack{m+n \in 2 \mathbb{Z}, m^{2}+n^{2}>0}} \frac{\left(z-\omega_{m n}-2\right)^{3}}{\left|\left(z-\omega_{m n}-2\right)^{3}-(\zeta-2)^{3}\right|^{2}} \\
& =\sum_{\substack{m+n \in 2 \mathbb{Z}, m^{2}+n^{2}>0}} \frac{\left(\overline{z_{1}-\omega_{m n}-2}\right)^{3}}{\left|\left(z_{1}-\omega_{m n}-2\right)^{3}-(\zeta-2)^{3}\right|^{2}} .
\end{aligned}
$$

Hence for $z \rightarrow \zeta_{0}$ on $\partial_{1} P^{+}$

$$
\partial_{\nu_{\zeta}} G_{1}(z, \zeta)=\frac{(\sqrt{3}-i)\left(z-z_{1}\right)}{|z-\zeta|^{2}}(1+o(1))
$$

On $\partial_{2} P^{+} \zeta=\zeta_{2}=\bar{\zeta}+2 i \sqrt{3}$ and $\zeta-i \sqrt{3}=\bar{\zeta}+i \sqrt{3}$, for $m=n=0$ in (22), the formula becomes

$$
\begin{aligned}
& -6 i(\zeta+1-i \sqrt{3})^{2} \\
& \cdot \frac{(z+1-i \sqrt{3})^{3}-(\overline{z+1-i \sqrt{3}})^{3}}{\left|(z+1-i \sqrt{3})^{3}-(\zeta+1-i \sqrt{3})^{3}\right|^{2}} .
\end{aligned}
$$

This term is not singular for $z \neq \zeta$ and the terms of the sum can be in general rewritten as $\left(z-\omega_{m n}+1-i \sqrt{3}\right)^{3}=\left(\overline{z_{1}}-\right.$ $\left.\omega_{k l}+1+i \sqrt{3}\right)^{3}$ for certain $k+l \in 2 \mathbb{Z}$. Therefore

$$
\begin{aligned}
& \sum_{m+n \in 2 \mathbb{Z}} \frac{\left(z-\omega_{m n}+1-i \sqrt{3}\right)^{3}}{\left|\left(z-\omega_{m n}+1-i \sqrt{3}\right)^{3}-(\zeta+1-i \sqrt{3})^{3}\right|^{2}} \\
& =\sum_{m+n \in 2 \mathbb{Z}}\left|\frac{\left(\overline{z_{1}-\omega_{m n}+1-i \sqrt{3}}\right)^{3}}{\left(z-\omega_{m n}+1-i \sqrt{3}\right)^{3}-(\zeta+1-i \sqrt{3})}\right|^{2} .
\end{aligned}
$$

Letting $z \rightarrow \zeta_{0}, z_{1} \rightarrow \zeta_{0} \in \partial_{1} P^{+}$the sum (22) tends to 0 .

Similarly, for the rest parts of the boundary $\partial_{3} P^{+}, \partial_{4} P^{+}$ one can get that the sums in (21) and (20) tend to zero as we let $z \rightarrow \zeta_{0} \in \partial_{1} P$. As a result for the case $\zeta_{0} \in \partial_{1} P^{+}$

$$
\begin{aligned}
\lim _{z \rightarrow \zeta_{0}} & {\left[-\frac{1}{4 \pi} \int_{\partial P^{+}} \gamma(\zeta) \partial_{\gamma_{\zeta}} G_{1}(z, \zeta) d s_{\zeta}\right] } \\
= & \lim _{z \rightarrow \zeta_{0}}\left[-\frac{(\sqrt{3}-i)}{4 \pi} \int_{\partial_{1} P^{+}} \gamma(\zeta) \frac{z-z_{1}}{|z-\zeta|^{2}} d s_{\zeta}\right] \\
= & \gamma\left(\zeta_{0}\right)
\end{aligned}
$$

on the boundary $\partial_{1} P$.

Case 2. Let $\zeta_{0}$ be from $\partial_{2} P^{+}$, where $\zeta_{0}=\overline{\zeta_{0}}+2 i \sqrt{3}, \zeta_{0}-i \sqrt{3}=$ $\overline{\zeta_{0}}+i \sqrt{3}$.

$$
\text { On } \partial_{2} P^{+}, \zeta=\bar{\zeta}+2 i \sqrt{3}, \zeta-i \sqrt{3}=\bar{\zeta}+i \sqrt{3} \text {. }
$$$$
\text { For } m=n=0 \text { the term in (22) is }
$$

$$
\begin{aligned}
& -6 i(\zeta+1-i \sqrt{3})^{2} \\
& -\frac{(z+1-i \sqrt{3})^{3}-(\overline{z+1-i \sqrt{3}})^{3}}{\left|(z+1-i \sqrt{3})^{3}-(\zeta+1-i \sqrt{3})^{3}\right|^{2}} .
\end{aligned}
$$


On this boundary $z=z_{2}=\bar{z}+2 i \sqrt{3}$ and $z-i \sqrt{3}=\bar{z}+i \sqrt{3}$ or $z_{2}-i \sqrt{3}=\bar{z}+i \sqrt{3}$; therefore

$$
\begin{gathered}
(z+1-i \sqrt{3})^{3}-(\overline{z+1-i \sqrt{3}})^{3}=(z+1-i \sqrt{3})^{3} \\
-\left(z_{2}+1-i \sqrt{3}\right)^{3}=\left(z-z_{2}\right)\left[(z+1-i \sqrt{3})^{2}\right.
\end{gathered}
$$

$$
\begin{aligned}
& +(z+1-i \sqrt{3})\left(z_{2}+1-i \sqrt{3}\right) \\
& \left.+\left(z_{2}+1-i \sqrt{3}\right)^{2}\right] .
\end{aligned}
$$

Substituting the latter into (34) and considering $z \rightarrow \zeta_{0} \in$ $\partial_{2} P^{+}, \zeta_{0} \neq 1+i \sqrt{3}, \zeta=\zeta_{0}$

$$
\lim _{z \rightarrow \zeta_{0}}\left\{\frac{-6 i(\zeta+1-i \sqrt{3})^{2}\left[(z+1-i \sqrt{3})^{2}+(z+1-i \sqrt{3})\left(z_{2}+1-i \sqrt{3}\right)+\left(z_{2}+1-i \sqrt{3}\right)^{2}\right]}{\left|(z+1-i \sqrt{3})^{2}+(z+1-i \sqrt{3})(\zeta+1-i \sqrt{3})+(\zeta+1-i \sqrt{3})^{2}\right|^{2}}\right\}
$$

gives

$$
-2 i \frac{\left(\zeta_{0}+1-i \sqrt{3}\right)^{4}}{|\zeta+1-i \sqrt{3}|^{4}}=-2 i, \quad \zeta_{0} \neq-1+i \sqrt{3}
$$

For $m \neq 0, n \neq 0$ by

$$
\begin{aligned}
& \sum_{\substack{m+n \in 2 \mathbb{Z}, m^{2}+n^{2}>0}} \frac{\left(z-\omega_{m n}+1-i \sqrt{3}\right)^{3}}{\left|\left(z-\omega_{m n}+1-i \sqrt{3}\right)^{3}-(\zeta+1-\sqrt{3})^{3}\right|^{2}} \\
& =\sum_{\substack{m+n \in 2 \mathbb{Z} Z \\
m^{2}+n^{2}>0}} \frac{\left(\overline{z_{2}-\omega_{m n}+1+i \sqrt{3}}\right)^{3}}{\left|\left(z_{2}-\omega_{m n}+1-i \sqrt{3}\right)^{3}-(\zeta+1-i \sqrt{3})^{3}\right|^{2}} .
\end{aligned}
$$

Letting $z \rightarrow \zeta_{0} \in \partial_{2} P^{+}$and since $z_{2} \rightarrow \zeta_{0}$, the sum tends to 0 . Then

$$
\partial_{\gamma_{\zeta}} G_{1}(z, \zeta)=-2 i \frac{z-z_{2}}{|z-\zeta|^{2}}(1+o(1))
$$

on $\partial_{2} P^{+}$. Similar computations on the boundary parts $\partial_{1} P^{+}$, $\partial_{3} P^{+}, \partial_{4} P^{+}$give that the sums (21) and (20) tend to zero as $z \rightarrow \zeta_{0}$. Therefore, on the boundary part $\partial_{2} P^{+}$for $\zeta_{0} \in \partial_{2} P^{+}$

$$
\begin{aligned}
\lim _{z \rightarrow \zeta_{0}} & {\left[-\frac{1}{4 \pi} \int_{\partial P^{+}} \gamma(\zeta) \partial_{\nu_{\zeta}} G_{1}(z, \zeta) d s_{\zeta}\right] } \\
\quad= & \lim _{z \rightarrow \zeta_{0}}\left[-\frac{1}{2 \pi i} \int_{\partial_{2} P^{+}} \gamma(\zeta) \frac{z-z_{2}}{|z-\zeta|^{2}} d s_{\zeta}\right]=\gamma\left(\zeta_{0}\right) .
\end{aligned}
$$

Case 3. Let $\zeta_{0}$ be defined on $\partial_{3} P^{+}$by $\zeta_{0}=-(1 / 2)(1-i \sqrt{3}) \overline{\zeta_{0}}-$ $3+i \sqrt{3}$.

On $\partial_{3} P^{+}$with $\zeta=\check{\zeta}_{1}=-(1 / 2)(1-i \sqrt{3}) \bar{\zeta}-3+i \sqrt{3},(\zeta+2)^{3}=$ $(\bar{\zeta}+2)^{3}$.

For $m=n=0$ in (21) the formula becomes

$$
-3(-\sqrt{3}+i)(\zeta+2)^{2} \frac{(z+2)^{3}-(\bar{z}+2)^{3}}{\left|(z+2)^{3}-(\zeta+2)^{3}\right|^{2}} .
$$

Since $\left(\check{z_{1}}+2\right)^{3}=(\bar{z}+2)^{3}$, then

$$
\begin{aligned}
& (z+2)^{3}-(\bar{z}+2)^{3}=(z+2)^{3}-\left(\check{z_{1}}+2\right)^{3} \\
& \quad=\left(z-\check{z_{1}}\right)\left[(z+2)^{2}+(z+2)\left(\check{z_{1}}+2\right)+\left(\check{z_{1}}+2\right)^{2}\right] .
\end{aligned}
$$

Letting $z \rightarrow \zeta_{0}, \check{z_{1}} \rightarrow \zeta_{0}$, and $\zeta=\zeta_{0}$ for the fraction

$$
\lim _{z \rightarrow \zeta_{0}} \frac{3(-\sqrt{3}+i)(\zeta+2)^{2}\left[(z+2)^{2}+(z+2)\left(\check{z_{1}}+2\right)+\left(\check{z_{1}}+2\right)^{2}\right]}{\left|(z+2)^{2}+(z+2)(\zeta+2)+(\zeta+2)^{2}\right|^{2}}=\frac{(-\sqrt{3}+i)\left(\zeta_{0}+2\right)^{4}}{\left|\zeta_{0}+2\right|^{4}}=(\sqrt{3}+i)
$$

For the other terms of $(21)\left(z-\omega_{m n}+2\right)^{3}=\left(\overline{z_{1}}-\omega_{k l}+2\right)^{3}$ and

$$
\begin{aligned}
& \sum_{\substack{m+n \in 2 \mathbb{Z}, m^{2}+n^{2}>0}} \frac{\left(z-\omega_{m n}+2\right)^{3}}{\left|\left(z-\omega_{m n}+2\right)^{3}-(\zeta+2)^{3}\right|^{2}} \\
& \quad=\sum_{\substack{m+n \in 2 \mathbb{Z}, m^{2}+n^{2}>0}} \frac{\left(\overline{z_{1}-\omega_{m n}+2}\right)^{3}}{\left.\mid \omega_{m n}+2\right)^{3}-\left.(\zeta+2)^{3}\right|^{2}} .
\end{aligned}
$$

Therefore

$$
\partial_{\nu_{\zeta}} G_{1}(z, \zeta)=(-\sqrt{3}-i) \frac{z-z_{3}}{|z-\zeta|^{2}}(1+o(1))
$$

for $z \rightarrow \zeta_{0} \in \partial_{3} P^{+}$. On the boundary parts $\partial_{1} P^{+}, \partial_{2} P^{+}, \partial_{4} P^{+}$ in the same manner we can prove that the sums (19), (22), and 
(20) tend to zero as $z \rightarrow \zeta_{0} \in \partial_{3} P^{+}$. Thus for this Case 3 is as follows:

$$
\begin{aligned}
\lim _{z \rightarrow \zeta_{0}} & {\left[-\frac{1}{4 \pi} \int_{\partial P^{+}} \gamma(\zeta) \partial_{\nu_{\zeta}} G_{1}(z, \zeta) d s_{\zeta}\right] } \\
\quad= & \lim _{z \rightarrow \zeta_{0}}\left[-\frac{(\sqrt{3}+i)}{4 \pi} \int_{\partial_{3} P^{+}} \gamma(\zeta) \frac{z-\check{z_{1}}}{|z-\zeta|^{2}} d s_{\zeta}\right] \\
= & \gamma\left(\zeta_{0}\right)
\end{aligned}
$$

on the boundary part $\partial_{3} P$.

Case 4 . Let $\zeta_{0}$ be from $\partial_{4} P^{+}$, where $\zeta_{0}=\zeta_{4}=\bar{\zeta}$.

Obviously, similar calculations on the boundary parts imply the related sums to be convergent to zero, except for the boundary part $\partial_{4} P^{+}$, where the boundary behavior is to be observed carefully.

On $\partial_{4} P^{+}$with $\zeta=\bar{\zeta}$ for $m=n=0$ in formula (20) we have

$$
6 i(\zeta-2)^{2} \frac{(z-2)^{3}-(\bar{z}-2)^{3}}{\left|(z-2)^{3}-(\zeta-2)^{3}\right|^{2}}
$$

Here $z=z_{4}=\bar{z}$; then term (47) is

$$
\begin{aligned}
6 i(\zeta-2)^{2} & \frac{\left(z-z_{4}\right)}{|z-\zeta|^{2}} \\
& \cdot \frac{(z-2)^{2}+(z-2)\left(z_{4}-2\right)+\left(z_{4}-2\right)^{2}}{\left|(z-2)^{2}+(z-2)(\zeta-2)+(\zeta-2)^{2}\right|^{2}}
\end{aligned}
$$

and taking the limit in the second fraction for $z \rightarrow \zeta_{0} \in \partial_{4} P^{+}$ and since $\zeta=\zeta_{0}$

$$
\begin{aligned}
& \lim _{z \rightarrow \zeta_{0}} 6 i(\zeta-2)^{2} \frac{(z-2)^{2}+(z-2)\left(z_{4}-2\right)+\left(z_{4}-2\right)^{2}}{\left|(z-2)^{2}+(z-2)(\zeta-2)+(\zeta-2)^{2}\right|^{2}} \\
& \quad=2 i \frac{\left(\zeta_{0}-2\right)^{4}}{\left|\zeta_{0}-2\right|^{4}}=2 i .
\end{aligned}
$$

Again, the terms of the sum (20) are rewritten and it follows that

$$
\begin{aligned}
& \sum_{\substack{m+n \in 2 \mathbb{Z}, m^{2}+n^{2}>0}} \frac{\left(z-\omega_{m n}-2\right)^{3}}{\left|\left(z-\omega_{m n}-2\right)^{3}-(\zeta-2)^{3}\right|^{2}} \\
& =\sum_{\substack{m+n \in 2 \mathbb{Z}, m^{2}+n^{2}>0}} \frac{\left(\overline{z_{4}-\omega_{m n}-2}\right)^{3}}{\left|\left(z_{4}-\omega_{m n}-2\right)^{3}-(\zeta-2)^{3}\right|^{2}} .
\end{aligned}
$$

If $z \rightarrow \zeta_{0}, z_{4} \rightarrow \zeta_{0} \in \partial_{4} P^{+}$, this sum (20) tends to 0 for $\zeta \in \partial_{4} P^{+}$. Thus on this boundary part

$$
\begin{aligned}
& \partial_{\nu_{\zeta}} G_{1}(z, \zeta)=2 i \frac{z-z_{4}}{|z-\zeta|^{2}}(1+o(1)), \\
& \lim _{z \rightarrow \zeta_{0}}\left[-\frac{1}{4 \pi} \int_{\partial P^{+}} \gamma(\zeta) \partial_{\nu_{\zeta}} G_{1}(z, \zeta) d s_{\zeta}\right] \\
& =\lim _{z \rightarrow \zeta_{0}} \frac{1}{2 \pi i} \int_{\partial_{4} P^{+}} \gamma(\zeta) \frac{z-\bar{z}}{|z-\zeta|^{2}} d s_{\zeta}=\gamma\left(\zeta_{0}\right)
\end{aligned}
$$

on the boundary $\partial_{4} P^{+}$. Thus, equality (24) for the function $\varphi(z)$ is valid.

In the next lemma the boundary behavior of the function $\varphi(\zeta)$ in the corner points $\pm 2, \pm 1+i \sqrt{3}$, is observed. It is shown that the continuity of the function is preserved at all the corner points which are treated as an intersection of two lines through the boundary parts.

Lemma 6. If $\gamma \in C\left(\partial P^{+} ; \mathbb{C}\right)$, then

$$
\begin{aligned}
& \lim _{z \rightarrow \zeta_{0} \in \partial P^{+}}\left\{-\frac{1}{4 \pi} \int_{\partial P^{+}}\left[\gamma(\zeta)-\gamma\left(\zeta_{0}\right)\right] \partial_{\nu_{\zeta}} G_{1}(z, \zeta) d s_{\zeta}\right\} \\
& =0, \quad \zeta_{0} \in\{ \pm 2, \pm 1+i \sqrt{3}\}, z \in P^{+} .
\end{aligned}
$$

The proof of this lemma is given in detail in [9]. We consider now the main theorem of this paper.

\section{Theorem 7. The Dirichlet problem fo the Poisson equation}

$$
\begin{gathered}
w_{z \bar{z}}=f \text { in } P^{+}, \\
w=\gamma \text { on } \partial P^{+}
\end{gathered}
$$

$$
\text { for } f \in L_{p}\left(P^{+} ; \mathbb{C}\right), 2<p, \gamma \in C\left(\partial P^{+} ; \mathbb{C}\right)
$$

is uniquely solvable in the Sobolev space $W^{2, p}\left(P^{+} ; \mathbb{C}\right) \cap$ $C\left(\overline{P^{+}} ; \mathbb{C}\right)$ by

$$
\begin{aligned}
w(z)= & -\frac{1}{4 \pi} \int_{\partial P^{+}} \gamma(\zeta) \partial_{\nu_{\zeta}} G_{1}(z, \zeta) d s_{\zeta} \\
& -\frac{1}{\pi} \int_{P^{+}} f(\zeta) G_{1}(z, \zeta) d \xi d \eta
\end{aligned}
$$

where $\zeta=\xi+i \eta$.

Proof. We need to prove that (54) is a solution of the Poisson equation in problem (53). The property of the Pompeiu operator $T f(z)=-(1 / \pi) \int_{D}(f(\zeta) /(\zeta-z)) d \xi d \eta$, described in $[13,14]$ as $\partial_{\bar{z}} T f(z)=f(z)$, provides a weak solution of $w_{z \bar{z}}$

$$
G_{1}(z, \zeta)=\log \left|\prod_{m+n \in 2 \mathbb{Z}} \frac{\left(\zeta-\omega_{m n}-2\right)^{3}-(\bar{z}-2)^{3}}{\left(\zeta-\omega_{m n}-2\right)^{3}-(z-2)^{3}}\right|^{2}
$$


and the derivative

$$
\begin{aligned}
& \partial_{z} G_{1}(z, \zeta)=\frac{3(z-2)^{2}}{(\zeta-2)^{3}-(z-2)^{3}} \\
& -\frac{3(z-2)^{2}}{(\bar{\zeta}-2)^{3}-(z-2)^{3}} \\
& +\sum_{\substack{m+n \in 2 \mathbb{Z}, m^{2}+n^{2}>0}}\left[\frac{3(z-2)^{2}}{\left(\zeta-\omega_{m n}-2\right)^{3}-(z-2)^{3}}\right. \\
& \left.-\frac{3(z-2)^{2}}{\left(\overline{\zeta-\omega_{m n}-2}\right)^{3}-(z-2)^{3}}\right] .
\end{aligned}
$$

In order to construct the Pompeiu-type operator we consider the following term:

$$
\begin{aligned}
& \frac{3(z-2)^{2}}{(\zeta-2)^{3}-(z-2)^{3}} \\
& \quad=\frac{1}{\zeta-z}+\frac{2(3-z)-\zeta}{(\zeta-2)^{2}+(\zeta-2)(z-2)+(z-2)^{2}}
\end{aligned}
$$

Define a function

$$
\begin{gathered}
\tilde{g}(\zeta, z)=\frac{2(3-z)-\zeta}{(\zeta-2)^{2}+(\zeta-2)(z-2)+(z-2)^{2}} \\
-\frac{3(z-2)^{2}}{(\bar{\zeta}-2)^{3}-(z-2)^{3}} \\
+\sum_{m+n \in 2 \mathbb{Z},}\left(\frac{3(z-2)^{2}}{\left(\zeta-\omega_{m n}-2\right)^{3}-(z-2)^{3}}\right. \\
\left.-\frac{3(z-2)^{2}}{\left(\bar{\zeta}+n^{2}>0\right.}\right)
\end{gathered}
$$

which is analytic with respect to $z \in P^{+}$; then $\partial_{z} G_{1} z$, zeta $=$ $1 /(\zeta-z)+\widetilde{g}(\zeta, z)$. Then, the equation $w_{z \zeta}$ is rewritten as

$$
\begin{aligned}
\partial_{z \bar{z}} & \left\{-\frac{1}{\pi} \int_{P^{+}} f(\zeta) G_{1}(z, \zeta) d \xi d \eta\right\} \\
& =\partial_{\bar{z}}\left\{-\frac{1}{\pi} \int_{P^{+}} f(\zeta)\left[\frac{1}{\zeta-z}+\widetilde{g}(\zeta, z)\right] d \xi d \eta\right\} \\
& =f(z) .
\end{aligned}
$$

This provides the solution to the differential equation in problem (53) in a weak sense. The boundary condition $w=\gamma$ on the boundary $\partial P^{+}$holds because of Lemmas 5 and 6 .

\section{Conflict of Interests}

The author declares that there is no conflict of interests regarding the publication of this paper.

\section{References}

[1] H. Begehr and T. Vaitekhovich, "Harmonic Dirichlet problem for some equilateral triangle," Complex Variables and Elliptic Equations, vol. 57, no. 2-4, pp. 185-196, 2012.

[2] H. Begehr and T. Vaitekhovich, "How to find harmonic green functions in the plane," Complex Variables and Elliptic Equations, vol. 56, no. 12, pp. 1169-1181, 2011.

[3] H. Begehr and T. Vaitekhovich, "Green functions, reections and plane parqueting," Eurasian Mathematical Journal (EMJ), vol. 1, no. 1, pp. 17-31, 2010.

[4] H. Begehr and T. Vaitekhovich, "The parqueting-reection principle for constructing Green functions," in Proceedings of the 7th International Workshop "Analytical Methods of Analysis and Differential Equations" (AMADE '12), pp. 11-20, Cambridge Scientific Publishers, Minsk, Belarus, September 2012.

[5] H. Begehr and T. Vaitekhovich, "Schwarz problem in lens and lune," Complex Variables and Elliptic Equations, vol. 59, no. 1, pp. 76-84, 2014.

[6] S. Burgumbayeva, Boundary value problems for tri-harmonic functions in the unit disc [Ph.D. thesis], Free University of Berlin, Berlin, Germany, 2009, http://www.diss.fu-berlin.de/diss/ receive/FUDISSthesis000000012636.

[7] E. Gartner, Basic complex boundary value problems in the upper half plane [Ph.D. thesis], Free University of Berlin, Berlin, Germany, 2006, http://www.diss.fu-berlin.de/diss/receive/ FUDISSthesis000000002129.

[8] B. Shupeyeva, Some basic boundary value problems for complex partial differentialequations in quarter ring and half hexagon [Ph.D. thesis], Free University of Berlin, Berlin, Germany, 2013, http://www.diss.fu-berlin.de/diss/receive/ FUDISSthesis000000094596.

[9] B. Shupeyeva, "Harmonic boundary value problems in a quarter ring domain," Advances in Pure and Applied Mathematics, vol. 3, no. 4, pp. 393-419, 2012.

[10] T. Vaitsiakhovich, Boundary value problems for complex partial differential equations in a ring domain [Ph.D. thesis], Free University of Berlin, Berlin, Germany, 2008, http://www.diss .fu-berlin.de/diss/receive/FUDISSthesis000000003859.

[11] I. N. Vekua, Generalized Analytic Functions, International Series of Monographs in Pure and Applied Mathematics, Pergamon Press, Oxford, UK, 1962.

[12] Y. F. Wang and Y. J. Wang, "Schwarz-type problem of nonhomogeneous Cauchy-Riemann equation on a triangle," Journal of Mathematical Analysis and Applications, vol. 377, no. 2, pp. 557-570, 2011.

[13] H. Begehr and T. Vaitekhovich, "Harmonic boundary value problems in half disc and half ring," Functiones et Approximatio Commentarii Mathematici, vol. 40, no. 2, pp. 251-282, 2009.

[14] Y. Wang, Boundary value problems for complex partial differential equations in fan shaped domains [Ph.D. thesis], Free University of Berlin, Berlin, Germany, 2011, http://www.diss .fu-berlin.de/diss/receive/FUDISSthesis000000021359. 


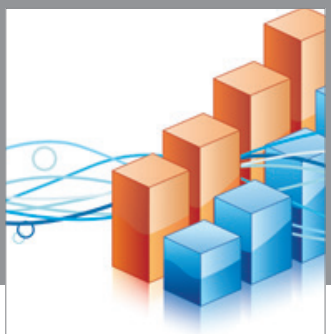

Advances in

Operations Research

vatem alat4

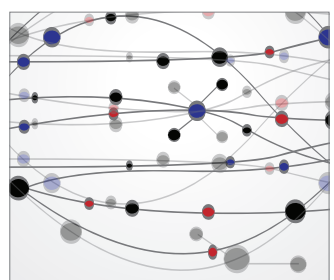

\section{The Scientific} World Journal
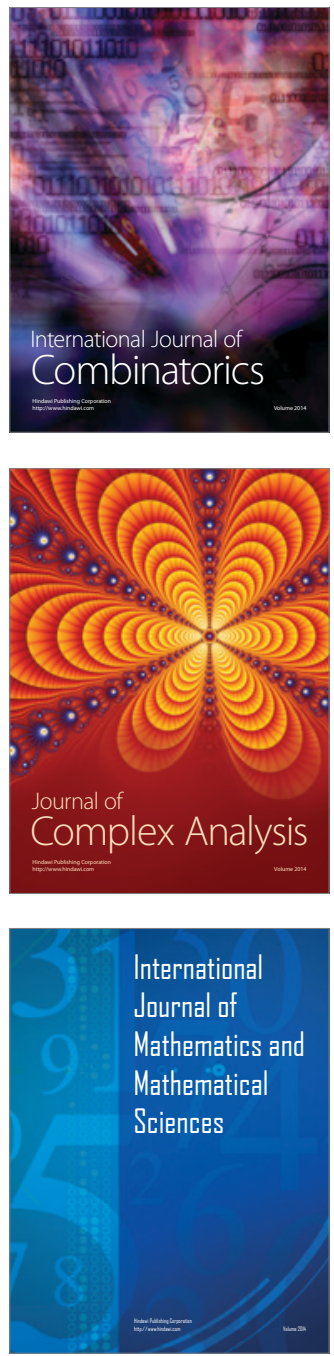
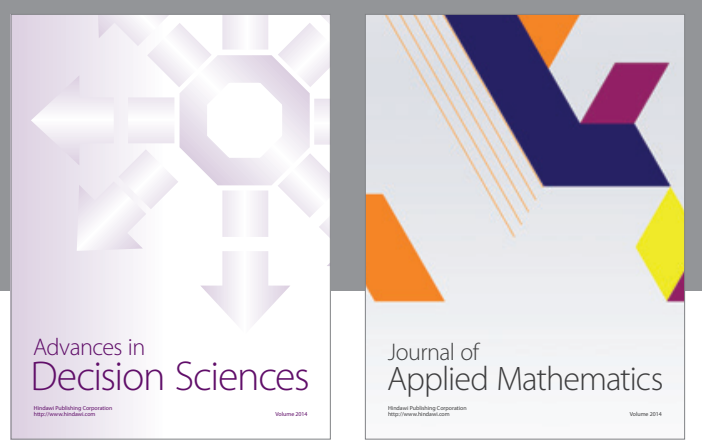

Algebra

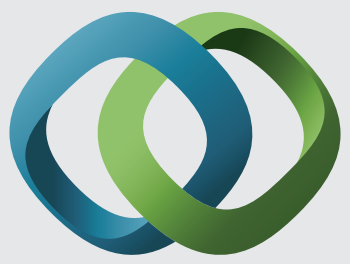

\section{Hindawi}

Submit your manuscripts at

http://www.hindawi.com
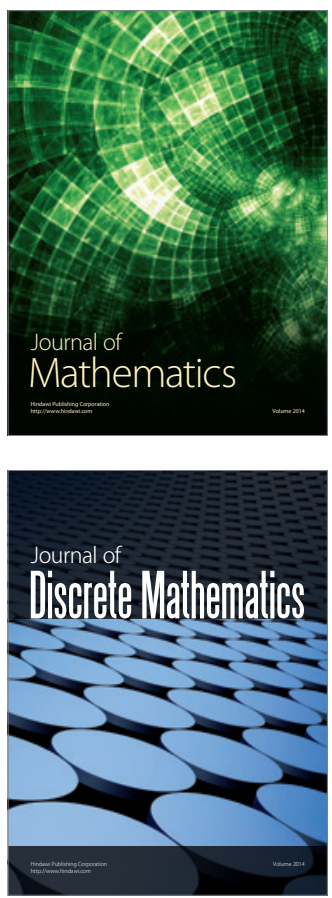

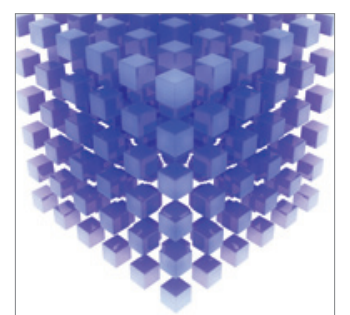

Mathematical Problems in Engineering
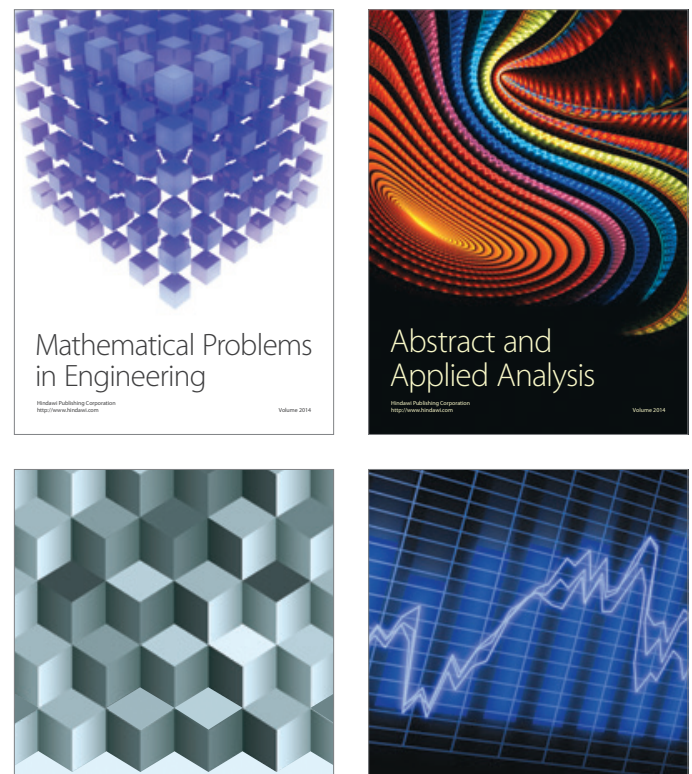

Journal of

Function Spaces

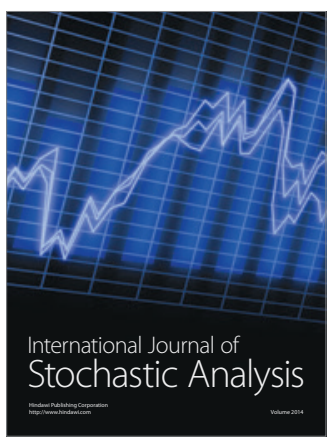

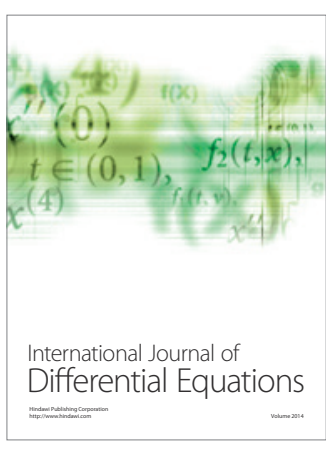
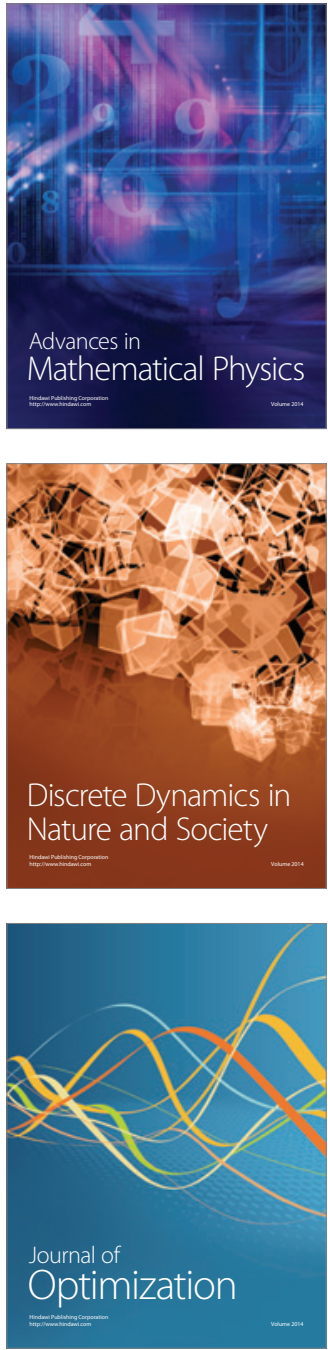